\title{
Risk factors of non-union in intramedullary stabilized diaphyseal long bone fractures: identifying the role of fracture stabilization strategies and concomitant injuries
}

\author{
Martijn Hofman ${ }^{1}$ (D) Hagen Andruszkow ${ }^{1} \cdot$ Frans L. Heyer $^{2} \cdot$ Philipp Kobbe $^{1} \cdot$ Frank Hildebrand $^{1} \cdot$ Martijn Poeze $^{2}$
}

Received: 5 November 2019 / Accepted: 18 February 2020 / Published online: 5 March 2020

(c) The Author(s) 2020

\begin{abstract}
Purpose Concomitant chest injury is known to negatively affect bone metabolism and fracture healing, whereas traumatic brain injury (TBI) appears to have positive effects on bone metabolism. Osteogenesis can also be influenced by the timing of fracture stabilization. We aimed to identify how chest injuries, TBI and fracture stabilization strategy influences the incidence of non-union.

Methods Patients with long bone fractures of the lower extremities who had been treated between 2004 and 2014 were retrospectively analysed. Non-union was defined as fracture healing not occurring in the expected time period and in which neither progression of healing nor successful union is expected without intervention. Diverse clinical and radiological parameters were statistically analysed using the Statistical Package for the Social Sciences (SPSS).

Results The total number of operations before consolidation was an independent predictor (odds ratio [OR] $=6.416$, $p<0.001)$ for the development of non-union in patients with long bone fractures. More specifically, patients treated according to the damage control orthopaedics (DCO) principle had a significantly higher risk of developing a non-union than patients treated according to the early total care $(\mathrm{ETC})$ principle $(\mathrm{OR}=7.878, p=0.005)$. Concomitant chest injury and TBI could not be identified as influencing factors for non-union development.

Conclusion Our results indicate that the number of operations performed in patients with long bone fractures should be kept as low as possible and that the indication for and the timing of DCO treatment should be meticulously noted to minimize the risk of non-union development.
\end{abstract}

Keywords Non-union $\cdot$ Treatment strategy $\cdot$ DCO $\cdot$ ETC $\cdot$ Chest injury $\cdot$ TBI

\section{Abbreviations}

AIS Abbreviated injury scale

AO Arbeitsgemeinschaft für osteosynthesefragen

ASA American College of Anaesthesiologists

ATLS $^{\circledR} \quad$ Advanced Trauma Life Support ${ }^{\circledR}$

Frank Hildebrand and Martijn Poeze contributed mutually.

$\begin{array}{ll}\text { CCI } & \text { Charlson comorbidity index } \\ \text { DCO } & \text { Damage control orthopaedics } \\ \text { ETC } & \text { Early total care } \\ \text { FHR } & \text { Fracture healing response } \\ \text { GCS } & \text { Glasgow coma scale } \\ \text { ISS } & \text { Injury severity score } \\ \text { OR } & \text { Odds ratio }\end{array}$

Martijn Poeze

m.poeze@mumc.nl

1 Department of Orthopedic Trauma and Reconstructive Surgery, University Hospital RWTH Aachen, Pauwelsstraße 30, 52074 Aachen, Germany

2 Division of Traumasurgery,, Department of Surgery, Maastricht University Medical Center, P. Debyelaan 25, 6229 HX Maastricht, The Netherlands 
SPSS Statistical package for the social sciences

TBI Traumatic brain injury

\section{Introduction}

Fracture healing depends on the interactions of many biomechanical and biological factors [1]. Disturbances in this process might result in non-union with an overall incidence of $1.5-10 \%$, increasing up to $40 \%$ in case of open fractures. In particular, non-unions of the lower extremities have been identified to significantly impair the post-traumatic quality of life [2], and have been associated with high direct and indirect costs $[3,4]$.

The risk factors for non-unions might either arise from injury characteristics, patient-specific factors, or from parameter associated with surgical fracture stabilization [5, 6]. Interactions between local and systemic inflammatory responses have been considered as the potential reasons for delayed fracture healing in chest trauma [6]. In contrast, TBI seems to be positively correlated with osteogenesis [7-9]. However, this association has not been found in all studies $[10,11]$. Furthermore, potential pathophysiological mechanisms for TBI-related impact on osteogenesis seem to be multifactorial (humoral, hormonal and cellular) and are far from clear [7, 12, 13].

Because of the enormous incapacitating effect of nonunions on the physical and mental health of patients, knowledge about the relevance of potential risk factors is of utmost importance. As the impact of several patient-(e.g. substance abuse, long-term use of steroids) and injury-specific (e.g. Gustilo type III open fracture) factors has already been well described [5], we focused on the influence of chest injury, TBI and fracture stabilization strategies in patients with long bone fractures of the lower extremities. Identifying the risk and predictive factors of non-union can help further develop prophylactic and therapeutic strategies for its treatment.

\section{Materials and methods}

\section{Study design and exclusion criteria}

We retrospectively analysed patients with diaphyseal femoral or tibial fractures who had been admitted and treated definitively with a reamed intramedullary locking nail at the Department of Orthopaedic Trauma and Reconstructive Surgery, University Hospital RWTH Aachen (Germany), or the Department of Traumasurgery, Maastricht University Medical Centre (The Netherlands) between 2004 and 2014. Clinical records and X-rays were retrieved for analysis. The patients' clinical course was followed until the last outpatient appointment. Patients who developed a non-union were placed in the NU group and those with normal fracture healing were placed in the control group. Non-union was defined as fracture healing not occurring in the expected time period and in which neither progression of healing nor successful union is expected without intervention.

To restrict the number of previously described factors influencing non-union development and to focus on chest injury, TBI and fracture stabilization strategy (ETC vs. DCO) as the influencing factors, we applied the following exclusion criteria: $17<$ age (years) $<80$, substance abuse (alcohol, tobacco, drugs), morbid obesity (BMI $>30$ ), mental disability, pregnancy, long-term use of steroids, bisphosphonates or thyroxin, lost to follow-up $<1$ year after trauma, Severe soft tissue damage (Gustilo and Anderson $>2$ ), Comminuted fracture, bone defect $>3 \mathrm{~cm}$, Pathological fractures, fractures of an adjacent joint, bilateral fractures, definitive treatment other than reamed intramedullary locking nail and primary definitive treatment elsewhere.

\section{Treatment algorithm}

All the patients were managed according to the principles of Advanced Trauma Life Support ${ }^{\circledR}\left(\right.$ ATLS $^{\circledR}$ ) and the S3 guidelines on the treatment of patients with severe injuries. For fracture treatment, patients underwent ETC with an antegrade intramedullary reamed locking nail, and if necessary, DCO at the earliest possible opportunity with an external fixator, which was subsequently converted to definitive osteosynthesis as soon as it was tolerated by the patient's clinical condition. Intravenous antibiotic prophylaxis was given in closed fractures as a single dose and for 3 days in open fractures. As soon as their clinical state allowed it, patients were mobilized with partial and consecutive increase to full weight-bearing, according to the fracture type. After discharge, the patients were seen in the outpatient clinic at 2 , $6,12,26$ and 52 weeks postoperatively. If union was not achieved at that time point, further controls took place until union was achieved or a revision was indicated.

\section{General health status and injury severity}

The general health status of the patients was estimated according to the American College of Anaesthesiologists (ASA) classification system and the Charlson comorbidity index (CCI) [14], which calculates an estimated relative risk of death based on the patient's age, cardiopulmonary and cerebrovascular condition, the presence of metabolic, gastrointestinal and infectious diseases as well as malignancies. Overall, injury severity was determined with the 2005 revised edition of the Abbreviated Injury Scale (AIS) and summarized to the Injury Severity Score (ISS) [15]. 


\section{Classification of chest injury}

Concomitant chest injuries were classified according to the $\mathrm{AIS}_{\text {thorax }}$, and patients were considered as having a concomitant chest injury when the $\operatorname{AIS}_{\text {thorax }}$ was $\geq 2$.

\section{Classification of TBI}

TBIs were classified according to their prehospital Glasgow coma scale (GCS) [16] and after computer tomography scanning. TBI was additionally classified according to the AIS $_{\text {head }}$. Patients were only considered as having a concomitant TBI when they had a prehospital GCS $\leq 12$ and an $\mathrm{AIS}_{\text {head }} \geq 2$.

\section{Fracture classification and fracture healing assessment}

Only patients with diaphyseal femoral (AO32.A-C) or tibial (AO42.A-C) fractures according to the AO (Arbeitsgemeinschaft für Osteosynthesefragen) classification system were included in the analysis. It was registered if these fractures were open (grade I or II according to Gustilo and Anderson) or closed.

Radiological imaging was reviewed and evaluated by two independent observers (HA and PK), who were blinded to concomitant injuries. A fracture was considered to be consolidated when both observers determined that three out of four cortices were bridged by a callus. Further, callus formation was quantified according to the fracture healing response described by Spencer [17].

\section{Outcome and complications}

In addition to our primary outcome parameter of nonunion, further neurological, cardiopulmonary, vascular, urinary tract, orthopaedic and systemic complications were registered.

\section{Statistical methods}

Data were analysed using SPSS (version 25; IBM Inc., Somers, NY, USA). Incidences are presented with counts and percentages, while continuous values are presented as mean \pm standard deviation. Differences between the groups were evaluated with Mann-Whitney's $U$ test for continuous data, and Pearson's $\chi^{2}$ test was used for categorical values. The nonparametric Spearman's rank test was used for statistical correlation. Multivariate logistic regression analysis was performed with non-union as the dependent variable to adjust for confounding variables. The results were reported as odds ratio with $95 \%$ confidence intervals (95\% CI). In general, a two-sided $p<0.05$ was considered to be statistically significant.

\section{Results}

\section{Demographic data}

A total of 136 and 68 patients were treated at the University Hospital RWTH Aachen (Germany) and the Maastricht University Medical Centre (The Netherlands), respectively. Of these, 100 patients (49.0\%) had femoral fractures and 104 (51.0\%) had tibial fractures. Overall, $25(12.3 \%)$ patients had a concomitant chest injury and $27(13.2 \%)$ had a concomitant TBI. A total of 98 patients $(48.0 \%)$ underwent ETC, and $106(52.0 \%)$ underwent DCO. Conversion to definitive osteosynthesis was performed $6.2 \pm 5.7$ days after trauma (Table 1).

\section{General health status, injury severity and clinical course}

The general health status and the injury severity, distribution and characteristics did not significantly differ between the two study groups. Over the clinical course, significant differences for the time period until definitive fracture stabilization $(p=0.012)$, the total number of operations performed before consolidation $(p<0.001)$ and the ratio of ETC to DCO $(p=0.016)$ were observed between the control and NU groups (Table 3).

\section{Nonparametric correlation analysis referring to non-union}

Non-union was diagnosed in $11.8 \%(n=24)$ of our patient population. Nonparametric correlation analysis showed a correlation between the fracture healing response and nonunion development $(r=-0.424, p<0.001)$. Also, the CCI was correlated with the incidence of non-union $(r=0.148$, $p=0.034)$. For TBI and chest trauma, no correlation was found (Table 4).

\section{Multivariate regression analysis referring to non-union}

The multivariate regression analysis referring to non-union showed that only the total number of operations before consolidation was an independent risk factor for non-union development $(\mathrm{OR}=6.416 ; p<0.001$; Table 2$)$.

Most patients underwent one or two operations and only seven patients underwent more than two operations, which were performed due to hardware failure $(n=3)$ or infection 
Table 1 Clinical and radiological parameters

\begin{tabular}{|c|c|c|c|}
\hline & $\begin{array}{l}\text { Control group } \\
(n=180)\end{array}$ & $\begin{array}{l}\text { NU group } \\
(n=24)\end{array}$ & $p$ value \\
\hline \multicolumn{4}{|l|}{ Clinical parameters } \\
\hline \multicolumn{4}{|l|}{ General } \\
\hline Age (years) & $35.5 \pm 15.5$ & $39.7 \pm 15.1$ & 0.167 \\
\hline Gender (female to male ratio) & 0.42 & 0.33 & 0.652 \\
\hline \multicolumn{4}{|l|}{ General health status } \\
\hline ASA classification system & $1.2 \pm 0.7$ & $1.3 \pm 0.7$ & 0.489 \\
\hline CCI & $0.6 \pm 1.3$ & $0.8 \pm 1.2$ & 0.334 \\
\hline \multicolumn{4}{|l|}{ Injury severity } \\
\hline ISS & $11.0 \pm 8.8$ & $10.7 \pm 7.5$ & 0.869 \\
\hline GCS & $14.2 \pm 2.4$ & $14.4 \pm 2.3$ & 0.622 \\
\hline AIS head & $0.4 \pm 0.9$ & $0.3 \pm 1.0$ & 0.753 \\
\hline AIS thorax & $0.4 \pm 1.0$ & $0.3 \pm 1.0$ & 0.735 \\
\hline Concomitant injuries & $54.2 \%$ & $50.0 \%$ & 0.699 \\
\hline AO classification & $\begin{array}{l}\text { A } 55.3 \% \\
\text { B } 36.3 \% \\
\text { C } 8.4 \%\end{array}$ & $\begin{array}{l}\text { A } 45.8 \% \\
\text { B } 37.5 \% \\
\text { C } 16.7 \%\end{array}$ & 0.385 \\
\hline Open/closed fracture & $\begin{array}{l}\text { Closed } 80.6 \% \\
\text { Open } 19.4 \%\end{array}$ & $\begin{array}{l}\text { Closed } 66.7 \% \\
\text { Open } 33.3 \%\end{array}$ & 0.117 \\
\hline \multicolumn{4}{|l|}{ Clinical course } \\
\hline Duration of hospital stay (days) & $17.1 \pm 17.6$ & $19.8 \pm 14.0$ & 0.535 \\
\hline In-hospital complications** & $23.3 \%$ & $20.0 \%$ & 0.785 \\
\hline Period trauma: first operative treatment (days) & $0.4 \pm 1.1$ & $0.6 \pm 1.9$ & 0.506 \\
\hline Period trauma-definitive osteosynthesis (days) & $3.9 \pm 5.3$ & $11.5 \pm 38.2$ & $0.012 *$ \\
\hline Period trauma-consolidation (days) & $326.5 \pm 278.3$ & - & \\
\hline Period definitive osteosynthesis-discharge & $15.9 \pm 17.4$ & $17.5 \pm 14.0$ & 0.663 \\
\hline Period definitive osteosynthesis-consolidation (days) & $322.2 \pm 277.5$ & - & \\
\hline Period trauma-discharge & $17.7 \pm 17.6$ & $19.8 \pm 14.0$ & 0.574 \\
\hline Total number of operations performed & $1.5 \pm 0.5$ & $2.1 \pm 0.8$ & $<0.001^{*}$ \\
\hline ETC vs. DCO & $\begin{array}{l}\text { ETC } 51.1 \% \\
\text { DCO } 48.9 \%\end{array}$ & $\begin{array}{l}\text { ETC } 25.0 \% \\
\text { DCO } 75.0 \%\end{array}$ & $0.016^{*}$ \\
\hline \multicolumn{4}{|l|}{ Radiological parameter } \\
\hline Fracture healing response & $1.5 \pm 0.3$ & $0.4 \pm 0.7$ & $<0.001 *$ \\
\hline Consolidation ( 3 out of 4 cortices) & $100 \%$ & $0 \%$ & \\
\hline
\end{tabular}

*Statistical significance, $p<0.05$

**Any secondary neurological, cardiopulmonary, vascular, urinary tract, orthopaedic and other complications were registered $(n=4)$ and not because of disturbed healing. Therefore, we focused on the patients who underwent either one or two operations. Of these patients, 96 underwent ETC and 88 of the 101 patients who had two operations $(87.1 \%)$ underwent DCO. Comparing these two subpopulations using non-parametric correlation, DCO strategy is more frequently applied with younger patients $(r=-0.277$, $p<0.001)$ and in male patients $(r=-0.208, p=0.005)$. Referring to injury-specific aspects, no correlation is found towards open fractures $(r=-0.017, p=0.815)$. Furthermore, patients with a poorer general health status (ASA: $r=0.162, p=0.028$ ) as well as patients with a more severe injury pattern (ISS: $r=0.471, p<0.001$ AIS $_{\text {head }}$ : $r=0.282, p<0.001 ; \mathrm{AIS}_{\text {thorax }}: r=0.308, p<0.001$; $\mathrm{AIS}_{\text {extremity }}: r=0.236, p=0.001$; concomitant injuries: $r=0.449, p<0.001$; complications: $r=0.314, p<0.001)$ were more likely to undergo DCO treatment. As described before, correlation between DCO treatment and the development of non-unions was found $(r=0.161, p=0.029)$ (Table 3).

In the multivariate regression analysis referring to nonunion in DCO vs. ETC, DCO represented an independent risk factor for non-union development with an odds ratio of $7.878(p=0.005$; Table 4$)$. 
Table 2 Multivariate regression analysis referring to nonunion analysing age, gender, ASA, CCI, ISS, GCS, AIS ${ }_{\text {head }}$, AIS $_{\text {thorax }}$, concomitant injuries, AO classification, open/closed fracture, period between trauma and definitive osteosynthesis and the total number of operations before consolidation as potential predictors (Nagelkerke: $R^{2}=0.294$ )

\begin{tabular}{|c|c|c|c|c|}
\hline Predictor & $\begin{array}{l}\text { Regression coef- } \\
\text { ficient }\end{array}$ & Odds ratio $(\mathrm{OR})$ & $\begin{array}{l}95 \% \text { confidence inter- } \\
\text { val }(95 \%-\mathrm{CI})\end{array}$ & $p$ value \\
\hline \multicolumn{5}{|l|}{ Patient-specific } \\
\hline Age (years) & 0.039 & 1.040 & $0.995-1.086$ & 0.083 \\
\hline Gender (male) & 0.203 & 1.225 & $0.358-4.186$ & 0.747 \\
\hline ASA & -0.070 & 0.933 & $0.404-2.152$ & 0.870 \\
\hline CCI & 0.017 & 1.017 & $0.616-1.679$ & 0.947 \\
\hline \multicolumn{5}{|l|}{ Injury-specific } \\
\hline ISS & -0.022 & 0.978 & $0.895-1.069$ & 0.624 \\
\hline GCS & 0.513 & 1.670 & $0.638-4.369$ & 0.296 \\
\hline $\mathrm{AIS}_{\text {head }}$ & 0.579 & 1.784 & $0.462-6.897$ & 0.401 \\
\hline $\mathrm{AIS}_{\text {thorax }}$ & -0.066 & 0.936 & $0.489-1.792$ & 0.842 \\
\hline Concomitant injuries & -0.236 & 0.790 & $0.235-2.661$ & 0.704 \\
\hline AO classification & 0.630 & 1.878 & $0.355-9.954$ & 0.459 \\
\hline Open/closed fracture & 0.651 & 1.917 & $0.571-6.440$ & 0.292 \\
\hline \multicolumn{5}{|l|}{ Treatment-specific } \\
\hline $\begin{array}{l}\text { Period trauma-definitive } \\
\text { osteosynthesis (days) }\end{array}$ & 0.042 & 1.043 & $0.995-1.094$ & 0.077 \\
\hline $\begin{array}{l}\text { Total number of opera- } \\
\text { tions before consolida- } \\
\text { tion }\end{array}$ & 1.859 & 6.416 & $2.434-16.910$ & $<0.001 *$ \\
\hline Constant & -14.309 & & & 0.058 \\
\hline
\end{tabular}

*Statistical significance, $p<0.05$
Table 3 Non-parametric correlation analysis of DCO treatment

\begin{tabular}{lcc}
\hline Parameter & $\begin{array}{l}\text { Correlation coef- } \\
\text { ficient }(r)\end{array}$ & $p$ value \\
\hline Age & -0.277 & $<0.001^{* *}$ \\
Gender & -0.208 & $0.005^{* *}$ \\
ASA & 0.162 & $0.028^{*}$ \\
ISS & 0.471 & $<0.001^{* *}$ \\
AIS head & 0.282 & $<0.001^{* *}$ \\
AIS thorax & 0.308 & $<0.001^{* *}$ \\
Concomitant injuries & 0.449 & $<0.001^{* *}$ \\
Complications & 0.314 & $<0.001^{* *}$ \\
AIS extremity & 0.236 & $0.001^{* *}$ \\
Open/closed fractures & -0.017 & 0.815 \\
Duration of hospital stay (days) & 0.729 & $<0.001^{* *}$ \\
Non-union & 0.161 & $0.029^{*}$ \\
\hline
\end{tabular}

*Statistical significance $p<0.05$

$* *$ Statistical significance $p<0.01$

\section{Discussion}

Non-unions of long bone fractures represent a challenging problem in trauma patients. Patient-, injury- and treatment-specific factors have been previously described to influence the occurrence of non-unions. Independent from the already well-known risk factors for non-union development, we aimed to focus on the impact of fracture stabilisation strategy, chest injury and TBI on the occurrence of non-unions in diaphyseal long bone fractures. Our main results can be summarized as follows:

1. The DCO fracture stabilisation strategy represents an independent risk factor for the development of nonunions in long bone fractures.

2. Chest injury and TBI were not identified as influencing factors for non-union development in diaphyseal long bone fractures.

Although DCO treatment is well accepted to be beneficial in certain subgroups of trauma patients, we found that this treatment strategy is associated with a higher risk of nonunion. Our findings were in accordance to those reported in the previous study by Rixen et al. [18]. In particular, the timing of conversion from external fixation to definitive stabilization has been suggested as an indispensable factor for non-union development $[19,20]$. In this context, late conversion ( $>10$ days after the initial treatment) has been associated with an increase in fracture-associated complications, such as non-union [21]. Therefore, it is of utmost importance to plan definitive surgery meticulously. In this context, Pape and Pfeifer revitalized the discussion on the DCO treatment strategy by introducing the concept of safe definitive surgery (SDS). In this concept, the time point of definitive fracture stabilization is based on a regular re-evaluation and 
Table 4 Multivariate regression analysis referring to nonunion analysing age, gender, ASA, CCI, ISS, GCS, AIS ${ }_{\text {head, }}$ AIS $_{\text {thorax }}$, concomitant injuries, AO classification, open/closed fracture, period between trauma and definitive osteosynthesis and DCO vs. ETC as potential predictors (Nagelkerke: $R^{2}=0.215$ )

\begin{tabular}{lclll}
\hline Predictor & $\begin{array}{l}\text { Regression coef- } \\
\text { ficient }\end{array}$ & Odds ratio (OR) & $\begin{array}{l}\text { 95\% confidence inter- } \\
\text { val (95\%-CI) }\end{array}$ & $p$ value \\
\hline Patient-specific & & & & \\
Age (years) & 0.036 & 1.037 & $0.990-1.086$ & 0.123 \\
Gender (male) & 0.406 & 1.501 & $0.424-5.313$ & 0.529 \\
ASA & -0.183 & 0.833 & $0.348-1.993$ & 0.681 \\
CCI & -0.007 & 0.993 & $0.568-1.736$ & 0.980 \\
Injury-specific & -0.020 & 0.980 & & 0.646 \\
ISS & 0.467 & 1.596 & $0.900-1.067$ & 0.316 \\
GCS & 0.500 & 1.648 & $0.446-6.094$ & 0.454 \\
AIS & & $0.493-1.769$ & 0.833 \\
AIS & -0.069 & 0.934 & $0.185-2.565$ & 0.578 \\
Concomitant injuries & -0.374 & 0.688 & $0.368-10.583$ & 0.427 \\
AO -classification & 0.680 & 1.974 & $0.577-7.219$ & 0.268 \\
Open/closed fracture & 0.714 & 2.042 & & 0.088 \\
Treatment-specific & & & $0.994-1.088$ & \\
Period trauma-definitive & 0.039 & 1.040 & $1.889-32.860$ & $0.005 *$ \\
$\quad$ osteosynthesis (days) & & & & 0.056 \\
DCO vs. ETC & 2.064 & 7.878 & & \\
Constant & -13.780 & & & \\
\hline
\end{tabular}

*Statistical significance, $p<0.05$ assessment of the patient's physiological condition and not on a suggested time point like in the DCO concept (e.g. not before day 5). The SDS concept; therefore, might lead to a dynamic combination of the advantages of both the DCO and ETC treatment strategy [22]. Our findings support the philosophy of this approach. The decision for DCO treatment strategy in patients with more severe injury and with poorer general health status, as demonstrated in our study, forms a gold standard nowadays. However, according to the SDS concept and taking our study results into consideration, approaches to identify patients who could potentially benefit from DCO should be improved to avoid the increased risk of non-union development.

To assess the relevance of concomitant injuries, we focused on chest injuries and TBI. However, both entities did not significantly influence the development of nonunions. However, it has to be noticed that the overall ISS of our study population was relatively low and the occurrence of chest or brain injuries in these patients is relatively infrequent, which made it especially difficult to demonstrate independent effects of concomitant injuries on fracture healing rates.

In contrast to our study, Recknagel et al. [23] suggest that chest trauma has a negative effect, particularly on the late phases of bone regeneration and fracture healing. A chest trauma-associated hypoxaemia-induced enhancement of local and systemic inflammation has been suggested as a potential pathomechanism by Kemmler et al. [24]. The differences between the results of our study and these experimental studies might be explained with different aspects. First, data obtained in animal experiments under standardized conditions might not be point-to-point transferable to the clinical situation with different confounding factors. Second, it has been postulated that the strategy for fracture fixation is an even more important factor for fracture healing than concomitant injuries [23]. This would be in line with our results and might explain why we did not observe an impact of chest trauma on the incidence of non-unions.

TBI did not have a significant influence on fracture healing. Therefore, the findings of this study are in contrast to the findings of the majority of studies that TBI has a positive influence on bone regeneration [11]. In this context, a retrospective study [8] found shorter healing time and increased callus dimensions in patients with concomitant TBI. In contrast to our study, they excluded patients treated according to the DCO principle. As fracture fixation represented an independent risk factor for disturbed fracture healing in our study, this might be one explanation for the different results of the studies. This assumption would also support the finding of the aforementioned experimental study that fracture fixation has more impact on fracture healing than concomitant injuries [23]. Another clinical study demonstrated shorter healing times, greater callus volumes and higher fracture healing rates in patients with concomitant TBI [25]. In contrast to our study, they included all long bones fractures (including humeral and fibular fractures) treated either with intramedullary nailing or plate osteosynthesis. Furthermore, they included only patients with severe 
TBI $(\mathrm{GCS}<8)$. These differences are likely to contribute to the differences between that study and our present study.

\section{Strength and limitations}

A strength of our study design is that by strict inclusion and exclusion criteria, we were able to analyse a specific patient cohort with smaller parameter variance and better comparability, in which we could focus on the influence of chest injury, TBI and fracture stabilisation strategy on non-union development by eliminating other possible confounding factors as much as possible.

One limitation of this study is its retrospective design. Second, a large number of patients (114) were lost to follow-up. Some of those patients may have had complications from the treatment and went for care elsewhere. On the other hand, patients with a straightforward healing process may have disengaged from the follow-up because they did not think it was essential. These phenomena could lead to possible selection bias.

Third, the treatment evaluated was limited to reamed intramedullary nailing and in consequence we cannot assess the influence of the studied parameter on non-unions in diaphyseal long bone fractures following other treatment strategies. However, reamed intramedullary nailing is an established technique, and is the preferred therapy for long bone shaft fractures of the lower extremities in adults [5].

\section{Conclusion}

Our results demonstrated that fracture stabilisation strategy is a far more powerful factor than concomitant injuries influencing non-union development in long bone fractures. Based on our finding that DCO stabilisation strategy is an eminent predictor for non-union development, it is of utmost importance in the clinical situation to critically review both: the indication for DCO and the time period until conversion to definitive treatment to minimize the risk of disturbed fracture healing. Our study further counterweights the rising evidence of concomitant chest injury predisposing and concomitant TBI protecting for non-unions in the specific situation of diaphyseal long bone fractures of the lower extremities. These findings could contribute to the improvement of the treatment principles and to the reduction of the treatment costs of non-union and its sequelae. Furthermore, reliably predicting the risk of non-union in certain fractures at the time of initial treatment would be a great advantage and could possibly modify treatment management.

Acknowledgements Open Access funding provided by Projekt DEAL.
Author contributions $\mathrm{MH}$ : conceptualization, methodology, validation, investigation, resources, data curation, writing - original draft preparation, reviewing and editing, visualization, project administration. HA: formal analysis, writing - reviewing and editing. FLH: investigation, resources, data curation. PK: conceptualization, methodology. FH and MP: writing - reviewing and editing, supervision. All authors read and approved the final submitted manuscript. Proofreading Scribendi Inc. (Chatham, Ontario N7M 0N3 Canada) performed the proofreading of our manuscript.

\section{Compliance with ethical standards}

Conflict of interest All authors declare that they have no conflict of interest.

Ethical approval All data in this study were obtained in accordance with the ethical standards of both institutional and/or national research committee and the guidelines of the revised United Nations declaration of Helsinki in 2013 (seventh revision) or comparable ethical standards.

Open Access This article is licensed under a Creative Commons Attribution 4.0 International License, which permits use, sharing, adaptation, distribution and reproduction in any medium or format, as long as you give appropriate credit to the original author(s) and the source, provide a link to the Creative Commons licence, and indicate if changes were made. The images or other third party material in this article are included in the article's Creative Commons licence, unless indicated otherwise in a credit line to the material. If material is not included in the article's Creative Commons licence and your intended use is not permitted by statutory regulation or exceeds the permitted use, you will need to obtain permission directly from the copyright holder. To view a copy of this licence, visit http://creativecommons.org/licenses/by/4.0/.

\section{References}

1. Giannoudis PV, Einhorn TA, Marsh D. Fracture healing: the diamond concept. Injury. 2007;38(Suppl 4):S3-6.

2. Tay WH, de Steiger R, Richardson M, et al. Health outcomes of delayed union and nonunion of femoral and tibial shaft fractures. Injury. 2014;45:1653-8.

3. Tzioupis C, Giannoudis PV. Prevalence of long-bone non-unions. Injury. 2007;38(Suppl 2):S3-9.

4. Giannoudis PV, Jones E, Einhorn TA. Fracture healing and bone repair. Injury. 2011;42:549-50.

5. Metsemakers WJ, Roels N, Belmans A, et al. Risk factors for nonunion after intramedullary nailing of femoral shaft fractures: remaining controversies. Injury. 2015;46:1601-7.

6. Hildebrand F, van Griensven M, Huber-Lang M, et al. Is there an impact of concomitant injuries and timing of fixation of major fractures on fracture healing? A focused review of clinical and experimental evidence. J Orthop Trauma. 2016;30:104-12.

7. Cadosch D, Gautschi OP, Thyer M, et al. Humoral factors enhance fracture-healing and callus formation in patients with traumatic brain injury. J Bone Joint Surg Am. 2009;91:282-8.

8. Giannoudis PV, Mushtaq S, Harwood P, et al. Accelerated bone healing and excessive callus formation in patients with femoral fracture and head injury. Injury. 2006;37(Suppl 3):S18-24.

9. Yang TY, Wang TC, Tsai YH, et al. The effects of an injury to the brain on bone healing and callus formation in young adults with fractures of the femoral shaft. J Bone Joint Surg Br. 2012;94:227-30. 
10. Morley J, Marsh S, Drakoulakis E, et al. Does traumatic brain injury result in accelerated fracture healing? Injury. 2005;36:363-8.

11. Hofman M, Koopmans G, Kobbe P, et al. Improved fracture healing in patients with concomitant traumatic brain injury: proven or not? Mediators Inflamm. 2015;2015:204842.

12. Song Y, Han GX, Chen L, et al. The role of the hippocampus and the function of calcitonin gene-related peptide in the mechanism of traumatic brain injury accelerating fracture-healing. Eur Rev Med Pharmacol Sci. 2017;21:1522-31.

13. Wildburger R, Zarkovic N, Tonkovic G, et al. Post-traumatic hormonal disturbances: prolactin as a link between head injury and enhanced osteogenesis. J Endocrinol Invest. 1998;21:78-86.

14. Charlson ME, Pompei P, Ales KL, et al. A new method of classifying prognostic comorbidity in longitudinal studies: development and validation. J Chronic Dis. 1987;40:373-83.

15. Baker SP, O'Neill B, Haddon W Jr, et al. The injury severity score: a method for describing patients with multiple injuries and evaluating emergency care. J Trauma. 1974;14:187-96.

16. Teasdale G, Jennett B. Assessment of coma and impaired consciousness. A practical scale. Lancet. 1974;2:81-4.

17. Spencer RF. The effect of head injury on fracture healing. A quantitative assessment. J Bone Joint Surg Br. 1987;69:525-8.

18. Rixen D, Grass G, Sauerland S, et al. Evaluation of criteria for temporary external fixation in risk-adapted damage control orthopedic surgery of femur shaft fractures in multiple trauma patients: "evidence-based medicine" versus "reality" in the trauma registry of the German Trauma Society. J Trauma. 2005;59:1375-94 (discussion 1394-1375).

19. Pape H, Stalp M, Griensven M, et al. Optimal timing for secondary surgery in polytrauma patients: an evaluation of 4,314 serious-injury cases. Der Chirurg; Zeitschrift fur alle Gebiete der operativen Medizen. 1999;70:1287-93.

20. Schuh A, Hausel M. Our experiences with change of osteosynthesis from external fixator to internal fixation in fractures of the lower limb. Zentralbl Chir. 2003;128:674-9.

21. Antich-Adrover P, Marti-Garin D, Murias-Alvarez J, et al. External fixation and secondary intramedullary nailing of open tibial fractures. A randomised, prospective trial. J Bone Joint Surg Br. 1997;79:433-7.

22. Pape HC, Pfeifer R. Safe definitive orthopaedic surgery (SDS): repeated assessment for tapered application of Early Definitive Care and Damage Control?: an inclusive view of recent advances in polytrauma management. Injury. 2015;46:1-3.

23. Recknagel S, Bindl R, Kurz J, et al. Experimental blunt chest trauma impairs fracture healing in rats. J Orthop Res. 2011;29:734-9.

24. Kemmler J, Bindl R, McCook O, et al. Exposure to $100 \%$ oxygen abolishes the impairment of fracture healing after thoracic trauma. PLoS ONE. 2015;10:e131194.

25. Khallaf FG, Kehinde EO, Hussein S. Bone healing and hormonal bioassay in patients with long-bone fractures and concomitant head injury. Medical Princ Pract. 2016;25:336-42. 